\title{
Hypolipidemic effect of XH601 on hamsters of Hyperlipidemia and its potential mechanism
}

\author{
Meng-Jie Zhao ${ }^{1}$, Shan-Shan Wang ${ }^{1}$, Yao Jiang ${ }^{1}$, Ying Wang ${ }^{1}$, Hong Shen ${ }^{1}$, Pei Xu ${ }^{2}$, Hua Xiang ${ }^{2}$ and Hong Xiao ${ }^{1 *}$ (D)
}

\begin{abstract}
Background: The novel compound XH601 is a synthesized derivative of formononetin. The present study was to investigate the hypolipidemia effect and potential mechanism of XH601.

Methods: Male Golden Syrian hamsters were induced by high-fat diet (HFD) for eight weeks and the hyperlipidemic model was established successfully. After XH601 treatment, serum and hepatic biochemistry parameters of hamsters were detected and the effect of XH601 on adipose tissue was also analyzed. Furthermore, 3 T3-L1 cell differentiation by Oil-Red-O staining was observed and the MRNA and protein expression of peroxisome proliferator-activated receptors (PPARs) were measured by qRT-PCR and Western-blot in mature adipocytes.

Results: The in vivo results suggest that XH601 significantly decreased the adipose weight and levels of serum triglyceride (TG), total cholesterol (TC), low-density lipoprotein (LDL-C), apolipoprotein B (Apo-B), apolipoprotein E (Apo-E), while increased serum high-density lipoprotein (HDL-C). The in vitro results implied that XH601 up-regulated the mRNA and protein expression of both PPARa and PPAR $\beta / \delta$ in a dose-dependent manner.

Conclusions: The study suggests that XH601 exhibited strong ability to improve the dyslipidemia in hamsters fed with high-fat diet. The potential mechanism of XH601 was associated with the up-regulation of PPARa and PPARB/ $\delta$ mRNA and protein expression.
\end{abstract}

Keywords: XH601, Hyperlipidemia, Golden Syrian hamster, 3 T3-L1 adipocyte, PPARs

\section{Background}

Cardiovascular diseases are the leading cause of both death and disability around the world [1] and hyperlipidemia is considered to be one of the greatest risk factors contributing to the prevalence and severity of atherosclerosis and subsequent coronary heart disease [2]. Hyperlipidemia is some kind of metabolic syndrome, due to dietary factors and a sedentary lifestyle, and is commonly characterized by elevated serum total cholesterol and triglycerides [3]. For over 20 years, statins have been utilized as one of the most widely prescribed medications for treatment of dyslipidemia [4]. Despite their highly beneficial effects in clinical practice,

\footnotetext{
*Correspondence: xhnkyy123@163.com

${ }^{1}$ Nanjing Medical University, Affiliated Nanjing Brain Hospital, No. 264 Guangzhou Road, Nanjing, Jiangsu 210029, People's Republic of China Full list of author information is available at the end of the article
}

most commonly seen side effects of statins have been reported, including mild-to-moderate elevations in liver transaminases [5] and other adverse effects including neurological damage, myopathy, and an increased risk of diabetes [6-9]. Recently, alternative therapeutics of herbs and natural agents are applied for patients with metabolic syndrome [10].

Over the past several decades, a pronounced increase of interest in the research of physiologic and pharmacologic effects of naturally bioactive compounds has taken place. Data obtained from preclinical animal experiments as well as clinical and epidemiological studies suggest that isoflavones may prevent dyslipidaemia, obesity, atherosclerosis, type 2 diabetes, nonalcoholic fatty liver disease, etc. [11-13] Formononetin is an O-methylated isoflavone present in different bean types at various levels. Previously, extracts containing formononetin have been 
investigated and are proved to have positive influences on dyslipidemia [14]. Peroxisome proliferatoractivated receptors (PPARs) are ligand-dependent factors that control energy homeostasis by modulating lipid and carbohydrate metabolism [15]. Research have determined the balanced PPARs activity of formononetin, which may enhance the attractiveness of formononetin in the treatment of metabolic syndrome [16].

The golden Syrian hamster has been increasingly utilized to do research on lipoprotein metabolism and to investigate the effects of hypolipidemic agents such as PPAR agonists, statins and CETP inhibitors [17] because of its similarity to human in terms of lipid utilization and high susceptibility to dietary cholesterol [18]. The main plasma cholesterol carrier of hamsters is LDL, and hamster LDL receptor gene has been isolated and characterized [19] by strong similarities to that of humans. Hamsters synthesize bile acids and hepatic cholesterol and also respond to dietary lipids like humans, thus they are prone to hypercholesterolemia induced by excessive dietary cholesterol intake, while rats are resistant to this [20]. Furthermore, hamsters resemble human beings in lipoprotein metabolism as well [21]. Hence, Syrian hamsters have been utilized for the observation of numerous pharmacologic agents with varying mechanisms of action [22].

The XH601 [3-(4-methoxyphenyl)-7-(undec-10-en-1yloxy)-4H-benzopyran-4-one] (Fig. 1), is a newly synthesized analogue of formononetin. It was provided by the Department of Pharmaceutical Chemistry, China Pharmaceutical University. In the present study, the hypolipidemic effect of XH601 was evaluated in vivo on a high-fat diet-induced hyperlipidemic hamster model [23]. Meanwhile, the potential mechanism was studied in vitro by detecting the relative mRNA and protein expression of PPARs in 3 T3-L1 adipocytes.

\section{Methods}

\section{Drug preparation}

The compound XH601 was provided by China Pharmaceutical University. The initial dose of atorvastatin (Lipitor, Pfizer) for humans is $10 \mathrm{mg}$ per day. The hamster dose was calculated by the human equivalent dose (HED) on the basis of body surface area: assuming a human weight of $60 \mathrm{~kg}$, the HED for 10 (mg)/60

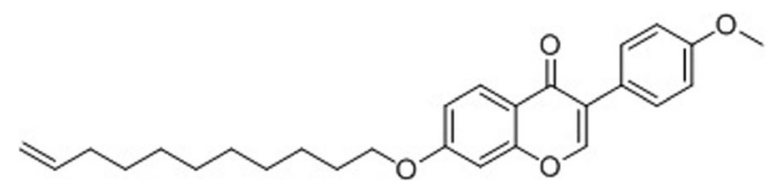

Fig. 1 Structural formula of XH601 $(\mathrm{kg})=0.17 \times 7.4=1.23 \mathrm{mg} / \mathrm{kg}$; the coefficient 7.4 was utilized to account for distinctions between hamster and human in body surface area [24, 25].

\section{Animals and treatment}

A total of 50 male Golden Syrian hamsters (90-100 g body weight) were purchased from Beijing Vital River Laboratory Animal Technology Co.,Ltd. (Beijing, China). After acclimation for one week, the hamsters were randomly divided into two groups: normal-fat diet (NFD) group $(n=10)$ and high-fat diet (HFD) group $(n=40)$. The HFD was composed of $10 \%$ lard, $2 \%$ cholesterol, $0.5 \%$ sodium deoxycholate and $87.5 \%$ regular diet. The food consumption was restricted to $10 \mathrm{mg} /$ day/hamster. After 8 weeks, the hyperlipidemic hamsters were subdivided into 4 groups ( $n=10$ in each group). (1) HFD group: HFD with vehicle (soybean oil) treatment; (2) ATO group: HFD with $1.23 \mathrm{mg} / \mathrm{kg} /$ day atorvastatin; (3) Low-dose XH601 group: HFD with $20 \mathrm{mg} / \mathrm{kg} /$ day XH601; (4) High-dose XH601 group: HFD with 50 mg/ $\mathrm{kg} /$ day XH601. The vehicle group received the same volume of solution equivalent to body weight. Vehicle and drugs were orally administered to hamsters by gastric intubation once a day for 8 weeks.

All animal experiments were approved by the Committee on the Medical Ethics of Nanjing Brain Hospital, affiliated to Nanjing Medical University (Permit Number: 2011KY45), and followed National Research Council Guidelines.

\section{Sample collection}

During the experimental period, body weights were recorded every two weeks. Blood from each hamster was collected from the retro-orbital sinus with a capillary tube after an over-night fasting. Serum was obtained after the blood was centrifuged $\left(8000 \mathrm{rpm}\right.$ at $4{ }^{\circ} \mathrm{C}$ for $15 \mathrm{~min}$ ) and stored at $-80{ }^{\circ} \mathrm{C}$ until analysis. The animals were sacrificed by cervical dislocation and tissues were harvested, weighed, snap frozen in liquid nitrogen and stored at $-80{ }^{\circ} \mathrm{C}$ until use.

\section{Serum and hepatic biochemistry parameters analysis}

The serum total cholesterol (TC), triglycerides (TG), high-density lipoprotein cholesterol (HDL-C), lowdensity lipoprotein cholesterol (LDL-C), apolipoprotein B (Apo-B), apolipoprotein E (Apo-E), aspartate aminotransferase (AST), alanine aminotransferase (ALT) and hepatic TC and TG were measured by enzymatic colorimetric methods using commercial kits (Nanjing Jiancheng Bioengineering Institute, Nanjing, China) following the manufacturer's instructions. 


\section{Histological analysis}

Liver and adipose tissues were isolated from hamsters, fixed in $10 \%$ formalin, and embedded in paraffin. Sections were abtained and later stained with hematoxylin and eosin (H\&E) for the histological examination. The frozen sections of liver were rinsed with distilled water, stained with $0.2 \%$ Oil-Red $\mathrm{O}$ and $60 \%$ isopropanol for $10 \mathrm{~min}$ at $37{ }^{\circ} \mathrm{C}$, and then rinsed again with distilled water. Tissue sections were then observed with a microscope.

\section{Culture of 3 T3-L1 preadipocytes and stimulation}

3 T3-L1 preadipocytes were obtained from Nanjing KeyGEN Biotech Co., Ltd. (Nanjing, China). The cells were cultured in Dulbecco's modified Eagle's medium (DMEM) supplemented with $10 \%$ fetal bovine serum (FBS) at $37^{\circ} \mathrm{C}$ in a humidified incubator under 5\% CO2. Adipocytic differentiation was induced by DMEM containing $10 \%$ FBS, $10 \mathrm{mg} / \mathrm{L}$ insulin, $0.5 \mu \mathrm{M}$ isobutylmethylxanthine and $1 \mu \mathrm{M}$ dexamethasone for $48 \mathrm{~h}$. The cells were then maintained in media supplemented with $10 \% \mathrm{FBS}$ and $10 \mathrm{mg} / \mathrm{L}$ insulin for another $48 \mathrm{~h}$, followed by incubating with drugs at different concentrations for 48 h. 3 T3-L1 mature adipocytes monolayers treated by different concentrations of XH601 $\left(1 \times 10^{-7}, 1 \times 10^{-6}\right.$, $1 \times 10^{-5}$ and $1 \times 10^{-4} \mathrm{M}$ ) were washed three times with phosphate-buffered saline (PBS) and fixed for $2 \mathrm{~h}$ with $3.7 \%$ formaldehyde in PBS. Oil-Red O $(0.5 \%)$ in isopropanol was diluted with $2 / 3$ volumes of water, filtered and added to the fixed cell monolayers for $2 \mathrm{~h}$ at room temperature. The cell monolayers were then washed with PBS, and the stained droplets in the cells were visualized. Image of the stained lipid droplets were collected on a microscope. Red staining reveals lipid droplets in the cytoplasm, indicating adipocyte differentiation.

\section{RNA isolation and quantitative real-time PCR}

3 T3-L1 adipocytes were incubated with atorvastatin $(10 \mu \mathrm{M})$ or XH601 at different concentrations (1 nM, $0.1 \mu \mathrm{M}$ and $10 \mu \mathrm{M})$ for $48 \mathrm{~h}$ before isolation of total RNA. Total RNA was isolated using Trizol Reagent. The RNA was reverse transcribed into cDNA using the Takara PrimeScript RT Master Mix (Code No. RR036A). The mRNA expression levels of PPARs were evaluated by qRT-PCR analysis using the FastStart Univeral SYBR Green Master (ROX) (Roche, Germany). qRT-PCR was performed on Eppendorf Mastercycler ep realplex (Eppendorf, Germany). The sequences for primers are listed in Table 1. Target mRNA expression in each sample was normalized to the housekeeping gene GAPDH. The $2^{-\Delta \Delta C t}$ method was used to calculate relative mRNA expression levels.
Table 1 The sequences of the primers used for real-time PCR

\begin{tabular}{ll}
\hline Gene name & Forward/reverse primers \\
\hline PPARa & 5'-TGAGGAAGCCGTTCTGTGAC-3' \\
& 5'-GGTGTCATCTGGATGGTTGC-3' \\
PPARß/ס & 5'-GCCTCGGGCTTCCACTAC-3' \\
& 5'-AGATCCGATCGCACTTCTCA-3' \\
PPARY & 5'-CCCCTGCTCCAGGAGATCTAC-3' \\
& 5'-GCAATCAATAGAAGGAACACGTGT-3' \\
&
\end{tabular}

\section{Western blot analysis}

Total proteins were extracted from the adipose tissue and adipocytes, using protein extraction kit (BestBio, Shanghai, China) and RIPA lysis buffer $(50 \mathrm{mM}$ Tris$\mathrm{HCl}, 150 \mathrm{mM} \mathrm{NaCl}, 1 \% \mathrm{NP}-40, \mathrm{pH}$ 7.4) containing 5\% PMSF, respectively. Protein concentrations were determined by BCA protein assay kit (Nanjing KeyGEN Biotech Co., Ltd., China). Equal amounts of proteins $(40 \mu \mathrm{g})$ were separated on 10\% SDS-polyacrylamide gel and transferred to PVDF membrane. After blocking in 5\% skim milk for $1 \mathrm{~h}$, the membranes were probed with primary antibodies (diluted 1:1000) against PPARs and $\beta$-Actin (Abcam, Cambridge, MA, USA) overnight at $4{ }^{\circ} \mathrm{C}$. Then the membranes were probed with secondary antibody coupled to horseradish peroxidase. Immunoreactive bands were visualized by using an enhanced chemiluminescence kit (Thermo) and were quantified using the Quantity One software (Bio-Rad Laboratories, UK).

\section{Statistical analysis}

Values are presented as the mean \pm S.E.M. Significant differences between diets or treatment groups were determined with SPSS 19.0 software (SPSS Inc., Chicago, IL, USA) using two-tailed Student t-test or one-way ANOVA with Dunnett's posttest. $P<0.05$ was considered statistically significant.

\section{Results}

Effect of XH601 on body mass of hamsters

The body mass of hamsters are in Table 2 . There were no differences in initial body weight and average daily food intake among all dietary groups in the experiment. After a 16-week induction, the body weights of hamsters in HFD group significantly increased $(P<0.05)$ comparing with those fed a normal diet (Fig. 2). Compared with the vehicle control, a significant decrease in the body weight was detected in the ATO group $(P<0.05)$ since drug administration, and XH601 at doses of $20 \mathrm{mg} / \mathrm{kg}$ and $50 \mathrm{mg} /$ $\mathrm{kg}$ resulted in a significant reduction in body weight gain of $31.18 \%(P<0.01)$ and $43.47 \%(P<0.01)$, respectively.

\section{Effect of $\mathrm{XH601}$ on serum lipid profiles}

As presented in the following figures, administration of hypercholesterolemic diet to hamsters for 8 weeks 
Table 2 Effect of XH601 on body mass of hamsters $(n=10)$

\begin{tabular}{llllll}
\hline Time point & NFD & HFD & XH601-L & XH601-H & ATO \\
\hline Week-0 & $104.95 \pm 3.13$ & $104.40 \pm 4.69$ & $103.76 \pm 3.59$ & $106.34 \pm 4.39$ & $105.81 \pm 4.09$ \\
2 & $118.88 \pm 4.89$ & $120.94 \pm 5.86$ & $122.74 \pm 6.56$ & $122.68 \pm 7.71$ & $119.14 \pm 8.53$ \\
4 & $123.95 \pm 5.30$ & $121.69 \pm 4.81$ & $123.42 \pm 7.83$ & $122.49 \pm 6.83$ & $120.28 \pm 7.33$ \\
6 & $124.90 \pm 4.69$ & $124.53 \pm 7.40$ & $123.87 \pm 7.09$ & $123.74 \pm 7.02$ & $122.76 \pm 8.86$ \\
8 & $128.92 \pm 3.16$ & $127.54 \pm 5.42$ & $127.97 \pm 7.51$ & $125.72 \pm 8.15$ & $125.71 \pm 9.94$ \\
10 & $128.61 \pm 2.04$ & $132.77 \pm 5.90$ & $128.92 \pm 5.49$ & $129.42 \pm 4.41$ & $125.64 \pm 8.75$ \\
12 & $129.84 \pm 6.13$ & $134.25 \pm 7.98$ & $129.61 \pm 5.24$ & $130.80 \pm 6.72$ & $124.00 \pm 2.94$ \\
14 & $129.81 \pm 4.89$ & $138.47 \pm 9.50$ & $130.95 \pm 6.25$ & $130.30 \pm 8.25$ & $124.20 \pm 7.23$ \\
16 & $126.70 \pm 4.48$ & $142.56 \pm 8.43^{\#}$ & $130.02 \pm 6.15^{*}$ & $127.92 \pm 7.61^{*}$ & $124.65 \pm 7.95^{*}$ \\
Weight gain & $21.75 \pm 6.09$ & $38.16 \pm 9.82^{*}$ & $26.26 \pm 4.72^{*}$ & $21.57 \pm 7.08^{* *}$ & $18.84 \pm 4.86^{* *}$ \\
\hline
\end{tabular}

${ }^{\#} P<0.05$ versus NFD; ${ }^{*} P<0.05,{ }^{* *} P<0.01$ versus HFD by one-way ANOVA with Dunnett's posttest

resulted in a profoundly elevation in serum TC, TG, LDL-C, HDL-C, Apo-B and Apo-E levels compared with the sham group, indicating that a hyperlipidemic hamster model had been induced successfully [26].

At eight weeks after administration of XH601 or ATO, serum TC and HDL-C levels significantly differed among groups, as shown in Fig. 3. TC concentrations were lower with ATO and XH601-H than with HFD alone by 45.54\% $(P<0.05)$ and $35.39 \%(P<0.05)$, respectively. And at the end of the experiment, serum HDL-C levels of XH601-L and XH601-H groups were higher than HFD group by 1.16 -fold $(P<0.05)$ and 1.19 -fold $(P<0.05)$, respectively.
As presented in Fig. 4, hamsters' serum TG concentration showed significant difference among experimental groups after 4-week administration of ATO or XH601. TG levels were lower with XH601-L and XH601-H than HFD group by $40.07 \%(P=0.001)$ and $37.91 \%(P=0.002)$. Compared with animals fed a high-cholesterol diet, XH601 at dose of $50 \mathrm{mg} / \mathrm{kg}$ significantly decreased serum LDL-C and Apo-B levels by $29.35 \%(P<0.05)$ and $28.25 \%$ $(P<0.05)$, respectively. At four weeks after drug administration, hamsters serum Apo-E levels of ATO, XH601-L and $\mathrm{XH601-H}$ groups declined than HFD group by $37.22 \%(P<0.05), 42.80 \%(P<0.05)$ and $44.18 \%$ $(P<0.01)$, respectively.

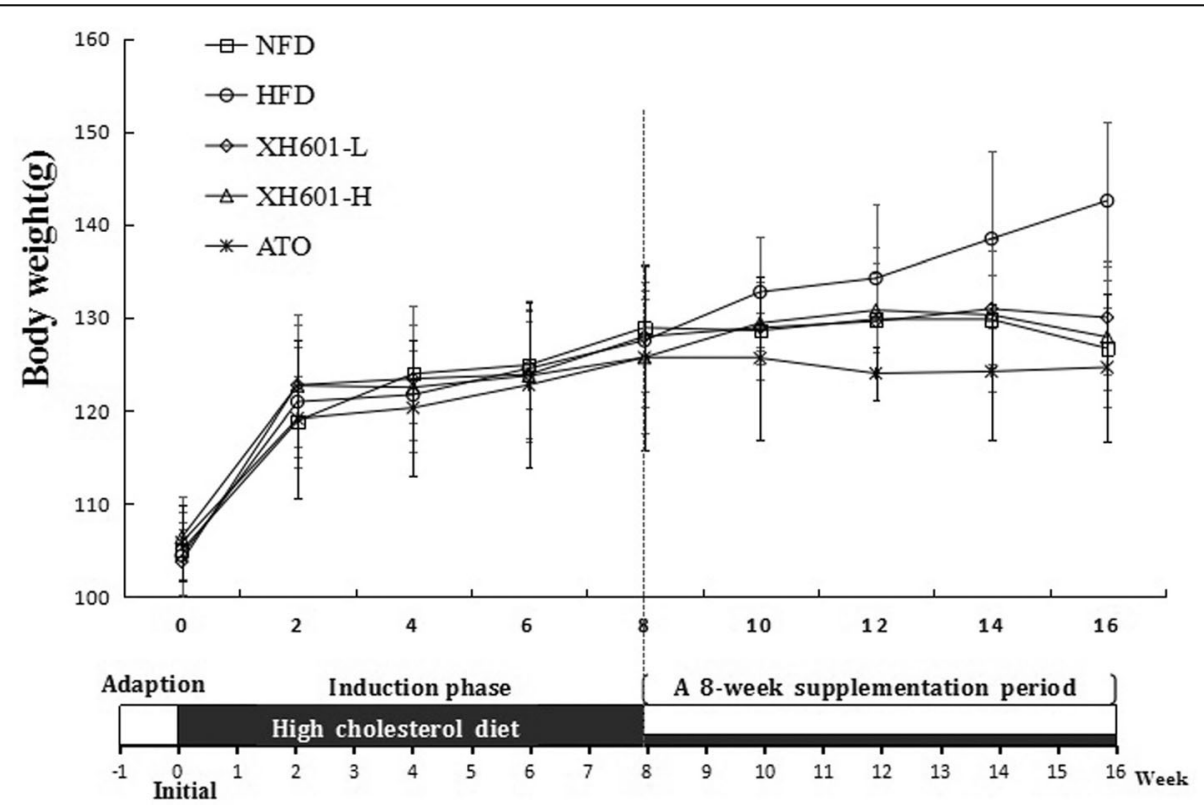

Fig. 2 Effect of XH601 on body mass of hamsters. In the induction phase, hamsters were separated into the NFD group ( $n=10)$ and HFD group $(n=40)$. After 8 weeks, the 40 hamsters were randomly assigned to four groups (ten hamsters/group) for solvent, XH601 (20 mg/kg/day or $50 \mathrm{mg} / \mathrm{kg} /$ day $)$ or ATO $(1.23 \mathrm{mg} / \mathrm{kg} /$ day $)$ administration 


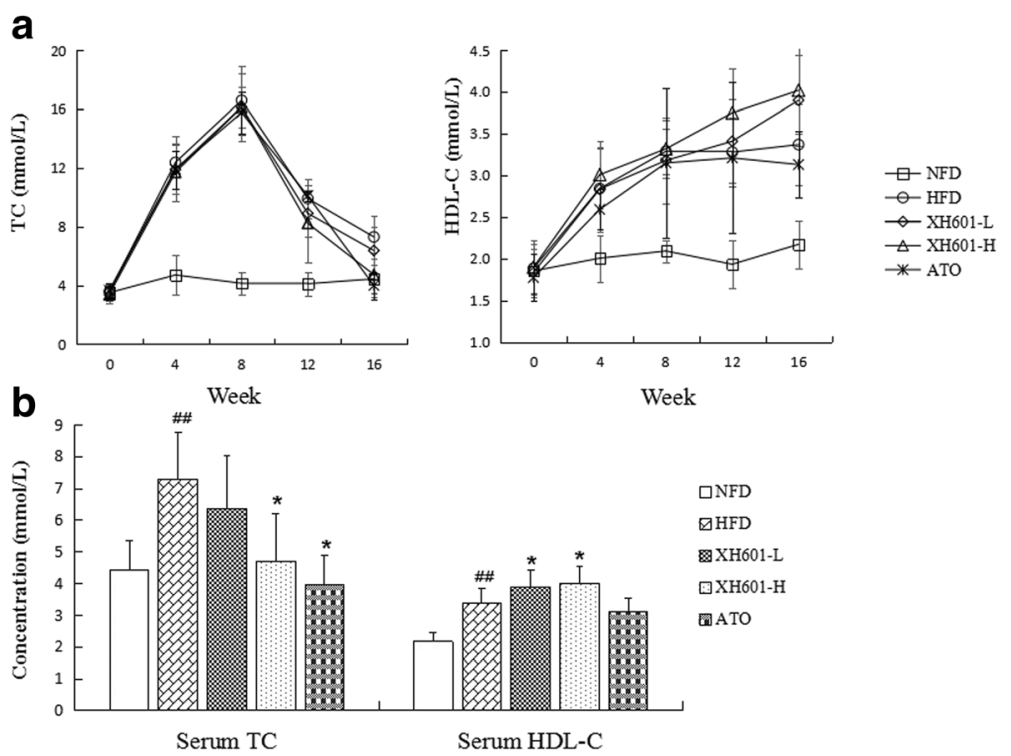

Fig. 3 Effect of eight-week XH601 treatment on lipid profiles. a Variations on serum TC and HDL-C levels in hyperlipidemic hamsters throughout the experiment. $\mathbf{b}$ Effect of eight-week drug treatment on serum TC and HDL-C levels. ${ }^{\# \#} P<0.01$ versus NFD; ${ }^{*} P<0.05$ versus HFD group by one-way ANOVA with Dunnett's posttest. TC: total cholesterol; HDL-C: high-density lipoprotein cholesterol

\section{Effect of XH601 on hepatic lipid levels}

Liver TC content significantly differed among groups and was higher with HFD alone, by 1.67 -fold $(P<0.05)$, than controls (Fig. 5a). Furthermore, hepatic TC level was lower with XH601-L and XH601-H than HFD by 38.33\% $(P<0.05)$ and $43.71 \%(P<0.05)$, respectively. Hepatic TG level of HFD group was pronounced increased comparing with NFC group, and XH601 at $50 \mathrm{mg} / \mathrm{kg} /$ day markedly declined the concentration of TG $(P<0.05)$.

\section{Effect of XH601 on hepatic dysfunction}

Concerning the side effects of statins on significantly elevating liver enzyme levels, we examined the serum AST and ALT concentrations of hamsters. As presented in Fig. 6, both AST and ALT levels of HFD group and ATO group obviously increased comparing to NFD group, while eight-week of low-dose XH601 administration $(20 \mathrm{mg} / \mathrm{kg})$ resulted in a profoundly decrease in AST than HFD group by $30.24 \%(P<0.05)$. Meanwhile, eight-week of XH601 treatment at dose of $50 \mathrm{mg} / \mathrm{kg}$ significantly declined AST and ALT levels by $40.28 \%$ $(P<0.01)$ and $45.20 \%(P<0.05)$, respectively.

\section{Effect of XH601 on adipose and liver tissue}

Hamsters were sacrificed after 8 weeks of XH601 administration; liver, kidney, total fat and epididymal fat pad (EFP) were removed and tissue weights were measured and calculated the percentages of the whole body weight individually. As shown in Table 3, the relative EFP, total fat and liver weight were marked higher in HFD group than control. The relative EFP weight was lower with XH601-L and XH601-H than HFD alone by $15.04 \%$ $(P<0.05)$ and $13.82 \%(P<0.05)$, respectively. Compared with the HFD group, the relative total fat weight of hamsters in XH601-L and XH601-H group significantly declined by $17.30 \%(P<0.05)$ and $16.82 \%(P<0.05)$. Meanwhile, the relative liver weight of hamsters in XH601-H group profoundly decreased by $16.87 \%$ $(P<0.05)$.

\section{Histological assessment}

In the liver section of the normal group, hepatic lobular architecture remained clear and intact without any abnormalities. Lipid deposits as macrovesicular and microvesicular steatosis were abundant in the section of the HFD group hamsters. It was shown that treatment with XH601 dose-dependently repressed these changes induced by high-fat diet and ameliorated the symptoms of fatty liver (Fig. 7b), with less lipid droplets in the Oil-Red $\mathrm{O}$ staining sections (Fig. 7a). While the histological pictures showed that liver tissue was damaged in ATO administrated hamsters. Meanwhile, histological analysis also showed that adipocyte size in adipose tissue was smaller in the XH601 groups than in the control group fed a high-fat diet (Fig. 7c).

\section{Effect of XH601 on adipocyte differentiation}

Differentiation of 3 T3-L1 preadipocytes can be induced into adipocytes in cell culture. They varied from an extended fibroblast-like morphology into a round one with cytoplasmic lipid vesicles containing newly 

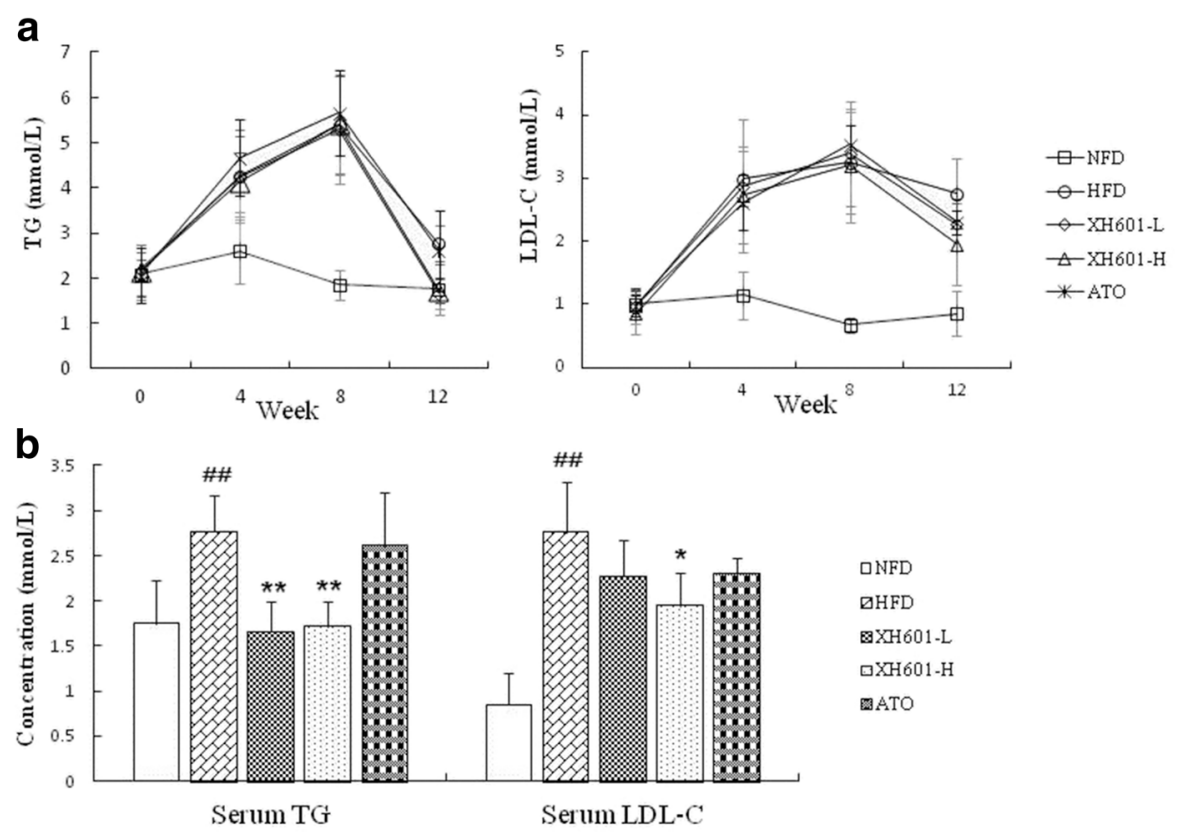

$\triangle N F D$

$\triangle \mathrm{HFD}$

$\mathrm{\otimes XH} 601-\mathrm{L}$

$\square \mathrm{XH} 601-\mathrm{H}$

MATO
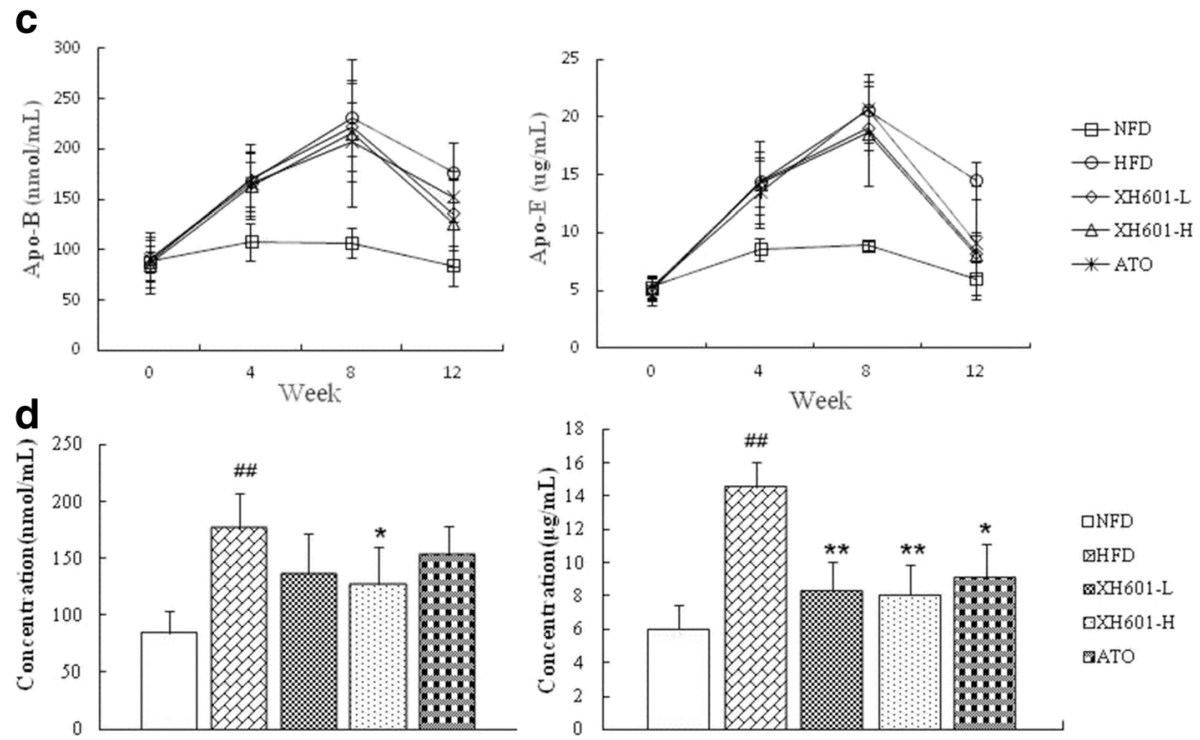

Fig. 4 Effect of four-week XH601 treatment on serum profiles. a Variations on serum TG and LDL-C levels in hyperlipidemic hamsters throughout the experiment. $\mathbf{b}$ Effect of four-week drug treatment on serum TG and LDL-C levels. $\mathbf{c}$ Variations on serum Apo-B and Apo-E levels in hyperlipidemic hamsters throughout the experiment. $\mathbf{d}$ Effect of four-week drug treatment on serum Apo-B and Apo-E levels. ${ }^{\# \#} P<0.01$ versus NFD; ${ }^{*} P<0.05$,

${ }^{* *} P<0.01$ versus HFD group by one-way ANOVA with Dunnett's posttest. TG: triglyceride; LDL-C: low-density lipoprotein cholesterol; Apo-B: apolipoprotein B; Apo-E: apolipoprotein E

biosynthesized triglyceride after differentiation. In this study, the effect of XH601 on adipogenic differentiation was investigated at four concentrations $\left(1 \times 10^{-7}, 1 \times 10\right.$ ${ }^{-6}, 1 \times 10^{-5}$ and $\left.1 \times 10^{-4} \mathrm{M}\right)$ in confluent 3 T3-L1 cells. Differentiation of 3 T3-L1 cell was characterized using Oil-Red-O staining for quantification through spectrophotometric analysis with isopropanol. As is shown in Fig. 8, lipid accumulation in 3 T3-L1 mature adipocytes significantly and dose-dependently decreased after drug treatment and XH601 showed the inhibitory effect on this adipogenic differentiation.

Effect of XH601 on PPARs mRNA and protein expression In this study, the hypolipidemic effects of XH601 in vivo has been determined, to further charify the mechanism of XH601, the effects of XH601 on PPARs expression were measured on $3 \mathrm{~T} 3-\mathrm{L} 1$ adipocytes in vitro. As presented in Fig. 9, atorvastatin significantly increased 

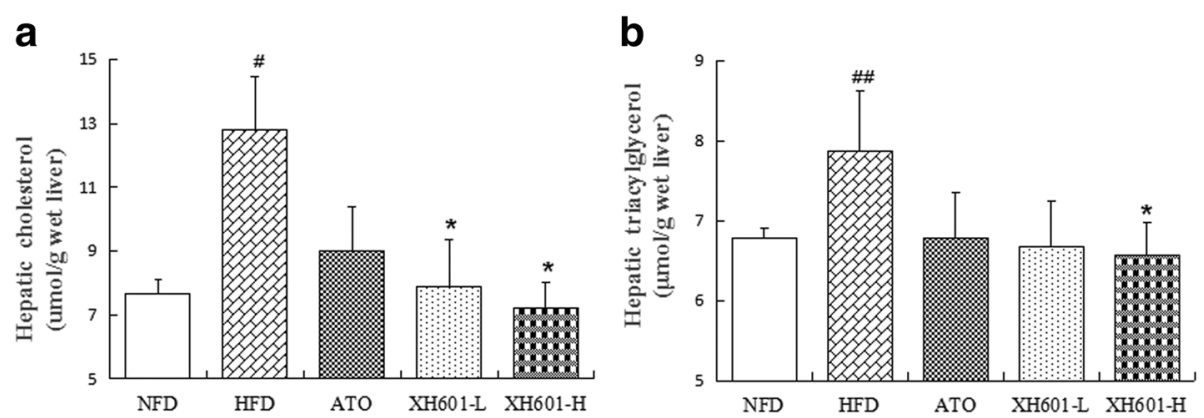

Fig. 5 Effect of XH601 on hepatic lipid levels. a Effect of eight-week drug treatment on hepatic TC levels. $\mathbf{b}$ Effect of eight-week drug treatment on hepatic TG levels. ${ }^{\#} P<0.05,{ }^{\# \#} P<0.01$ versus NFD; ${ }^{*} P<0.05$ versus HFD group by one-way ANOVA with Dunnett's posttest. TC: total cholesterol; TG: triglyceride

PPAR $\alpha$ PPAR $\beta / \delta$ and PPAR $\gamma$ mRNA expression by $2.6-$, 4.71- and 1.72-fold than control $(P<0.01)$, respectively. Compared to the control, XH601 profoundly up-regulated the mRNA expression of both PPAR $\alpha$ and PPAR $\beta /$ $\delta(P<0.01)$ in a dose-dependent manner, while showed no obvious effect on PPARy.

As shown in Fig. 10 and Fig. 11, the protein expression of PPAR $\beta / \delta$ and PPAR $\gamma$ after atorvastatin treatment was significantly increased. By contrast, the protein expression of PPAR $\alpha$ was profoundly up-regulated by highdose XH601 treatment $(P<0.05)$, while XH601 markedly improved PPAR $\beta / \delta$ protein expression $(P<0.01)$. The effects of XH601 on the protein expression of PPARs were roughly in accordance with its effect on the mRNA expression of them.

\section{Discussion}

The clinically significant consequences of hyperlipidemia include two life-threatening conditions, pancreatitis and atherosclerosis. Atherosclerosis is responsible for a large proportion of cardiovascular diseases, which are the leading cause of death in industrialized countries [26]. Additionally, many metabolic diseases, such as obesity and diabetes, are comorbidities associated with dyslipidemia. Drugs that attenuate dyslipidemia are acutely important in the prevention of cardiovascular diseases [27]. By activating PPARs system, statins have been advocated to play a major role in stabilization and regression of lipid-rich plaques [28-30]. However, the adverse effects of these drugs limit their more widely using and, thus, discovery and characterization of novel scaffolds based small molecules are expected to provide a superior profile in dyslipidemia intervention [20].

In the present study, we demonstrated the hypolipidemic properties and mechanism of XH601 modified from formononetin for the first time. The significant lipid-lowering effects of XH601 were observed in serum and hepatic lipid parameters. Moreover, our data indicated that XH601 decreased the weight of total adipose tissue, while atorvastatin not. To correspond to the hypolipidemic effects of XH601 on hamsters, its potential mechanism was also investigated in vitro [31]. Adipocytes play a significant role in lipid homeostasis and energy balance by triglyceride storage and free fatty acids release. In addition, adipocytes secrete dozens of factors which participate in energy metabolism of adipose tissues [32]. Thus, we used 3 T3-L1 adipocytes to explore the effects of XH601 on adipogenesis. Oil red O stain of mature differentiated 3 T3-L1 cells indicated that XH601 reduced the accumulation of lipid droplets
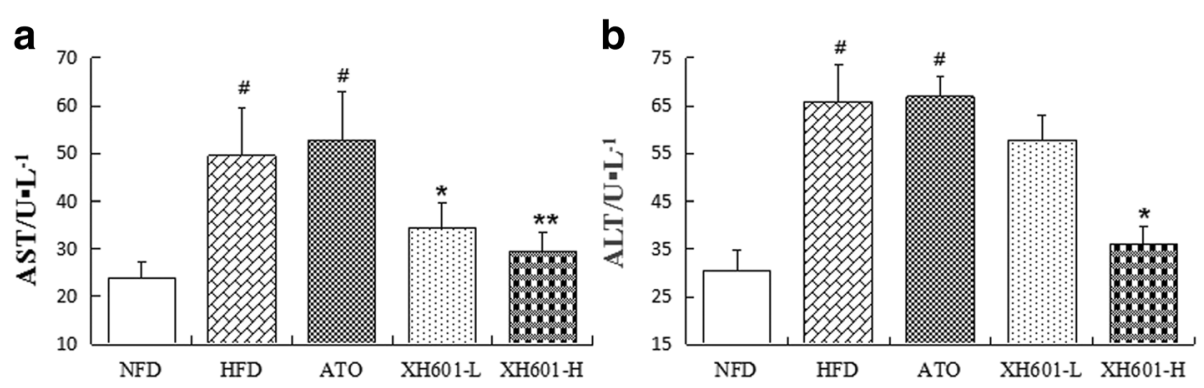

Fig. 6 Effect of XH601 on hepatic dysfunction. a Effect of eight-week drug treatment on serum AST levels. b Effect of eight-week drug treatment on serum ALT levels. ${ }^{\#} P<0.05$ versus NFD; ${ }^{*} P<0.05,{ }^{* *} P<0.01$ versus HFD group by one-way ANOVA with Dunnett's posttest. AST: aspartate aminotransferase; ALT: alanine aminotransferase 
Table 3 Tissue weights of hamsters at the end of the experiment $(n=10)$

\begin{tabular}{llllll}
\hline Tissue Weights & NFD & HFD & ATO & XH601-L & XH601-H \\
\hline EFP (g) & $2.10 \pm 0.54$ & $3.47 \pm 0.42^{\# \#}$ & $2.34 \pm 0.61^{* *}$ & $2.57 \pm 0.35^{* *}$ & $2.54 \pm 0.41^{* *}$ \\
Relative EFP (\%) & $1.68 \pm 0.34$ & $2.46 \pm 0.17^{\# \#}$ & $2.00 \pm 0.22^{*}$ & $2.09 \pm 0.23^{*}$ & $2.12 \pm 0.25^{*}$ \\
Total fat (g) & $3.19 \pm 0.85$ & $5.70 \pm 1.33^{\# \#}$ & $4.68 \pm 1.13$ & $4.47 \pm 0.59^{*}$ & $4.32 \pm 0.62^{*}$ \\
Relative fat (\%) & $2.55 \pm 0.57$ & $4.22 \pm 0.55^{\# \#}$ & $4.00 \pm 0.36$ & $3.49 \pm 0.52^{*}$ & $3.51 \pm 0.59^{*}$ \\
Liver (g) & $4.46 \pm 0.45$ & $5.61 \pm 0.70^{\#}$ & $5.59 \pm 0.54$ & $4.77 \pm 0.68$ & $4.63 \pm 0.93^{*}$ \\
Relative liver (\%) & $3.60 \pm 0.41$ & $4.03 \pm 0.46^{\#}$ & $4.87 \pm 0.51$ & $3.68 \pm 0.37$ & $3.35 \pm 0.28^{*}$ \\
Kidney (g) & $0.86 \pm 0.04$ & $0.85 \pm 0.09$ & $0.96 \pm 0.05$ & $0.84 \pm 0.06$ & $0.90 \pm 0.13$ \\
Relative kidney (\%) & $0.70 \pm 0.03$ & $0.61 \pm 0.02$ & $0.84 \pm 0.17^{* *}$ & $0.64 \pm 0.03$ & $0.68 \pm 0.06$ \\
\hline
\end{tabular}

${ }^{\#} P<0.05,{ }^{\# \#} P<0.01$ versus NFD; ${ }^{*} P<0.05,{ }^{* *} P<0.01$ versus HFD by one-way ANOVA with Dunnett's posttest. EFP: Epididymal fat pad
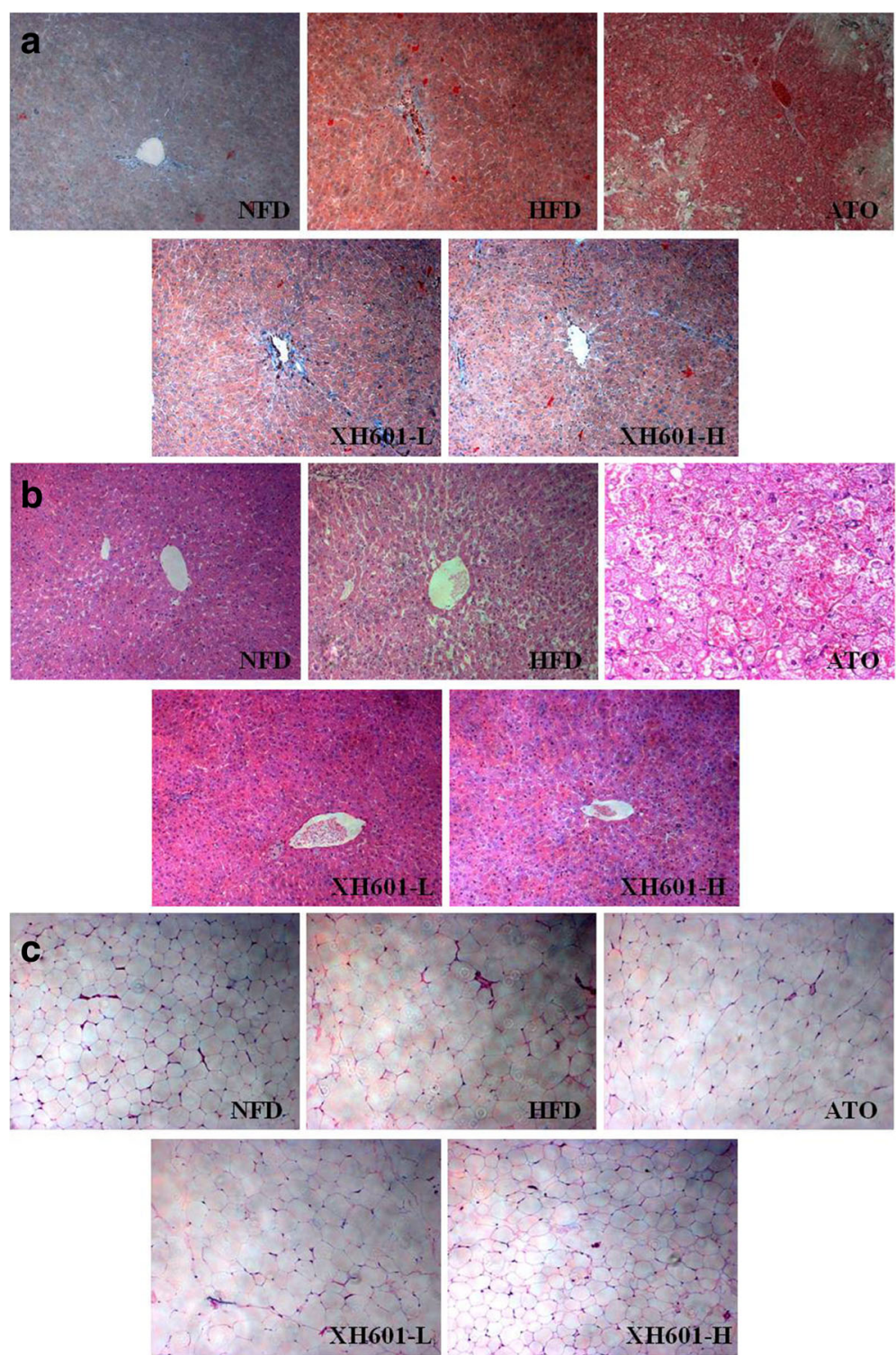

Fig. 7 Effect of XH601 on histology of liver and adipose tissue of hamsters (200X). $\mathbf{a}$ and $\mathbf{b}$ Photomicrograh of liver examination based on Oil-Red $\mathrm{O}$ staining and H\&E staining, respectively. c Photomicrograh of adipose tissue examination based on H\&E staining 


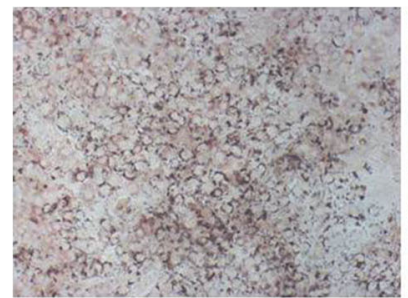

Control

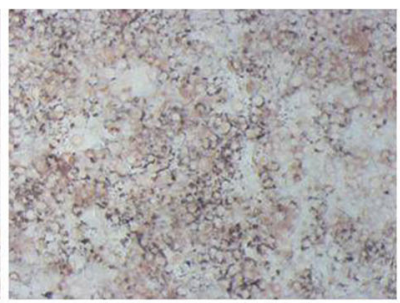

XH601 $1 \times 10^{-7} \mathrm{M}$

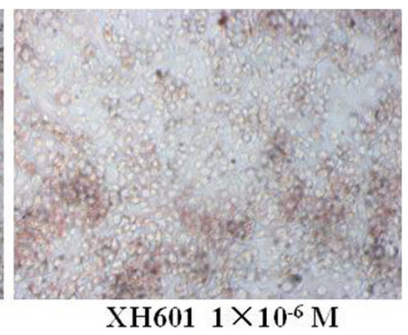

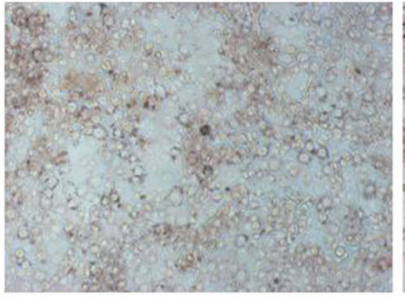

XH601 $1 \times 10^{-5} \mathrm{M}$

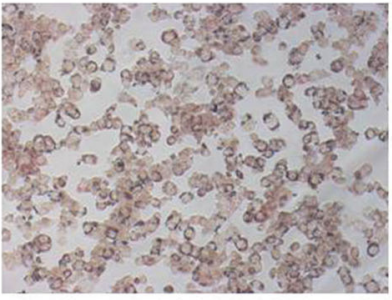

XH601 $1 \times 10^{-4} \mathrm{M}$

Fig. 8 Effect of XH601 on adipocyte differentiation. 3 T3-L1 mature adipocytes monolayers were treated by different concentrations of XH601 $\left(1 \times 10^{-7}\right.$, $1 \times 10^{-6}, 1 \times 10^{-5}$ and $1 \times 10^{-4} \mathrm{M}$ ) for $48 \mathrm{~h}$ and were fixed and stained with the lipophilic dye Oil-Red-O. Lipid droplets were visualized by Oil-Red-O staining

and decreased lipid content. These results showed that XH601 may play a positive role in the prevention of adipogenesis.

Considerable research effort has focused on isoflavones as the main hypolipidemic agent in soy because of their antioxidative and mild estrogenic activity. One relevant mechanism may be by PPARs, nuclear receptors that participate in cellular lipid homeostasis and insulin action [33]. PPARs are also transcriptional activators of genes encoding enzymes to metabolize fatty acid in the liver as well as other metabolic tissues [34] and atorvastatin has been demonstrated to exert cardiac protective influences by pronouncedly up-regulating PPARs expression [35-37]. In our research, relative protein expression of PPARs and phosphorylated PPARs (Additional file 1) were observed in 3 T3-L1 adipocytes and adipose tissues of hamsters, thereby investigating the potential mechanism of XH601.

PPAR $\alpha$ is a transcription factor that regulates the metabolism of lipids, carbohydrates, and amino acids and is activated by ligands such as drugs used to treat dyslipidemia [38]. By elevating lipoprotein lipase (LPL), triglyceride-VLDL lipolysis was increased and VLDL clearance was improved. Thereby atherogenic LDL-C, serum and hepatic TG were reduced. In addition, energy uncoupling was simultaneously induced in adipose tissue [20]. We found that XH601 activated PPAR $\alpha$ more efficiently than atorvastain in adipocytes. Correspondingly, in the high-fat diet-induced hyperlipidemic hamsters, 4-week treatment with XH601 significantly lowered

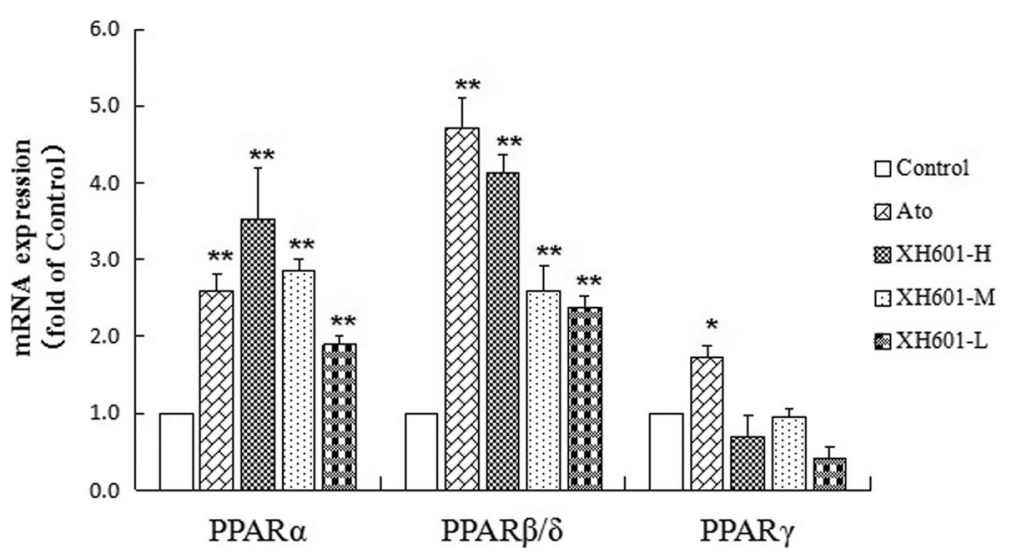

Fig. 9 Effect of XH601 on PPARs mRNA expression in 3 T3-L1 adipocytes. ${ }^{*} P<0.05,{ }^{* *} P<0.01$ versus control by one-way ANOVA with Dunnett's posttest. ATO: atorvastatin treatment at $10 \mu \mathrm{M}$ in cells; XH601-H: XH601 treatment at $10 \mu \mathrm{M}$ in cells; XH601-M: XH601 treatment at $0.1 \mu \mathrm{M}$ in cells; XH601-L: XH601 treatment at $1 \mathrm{nM}$ in cells 


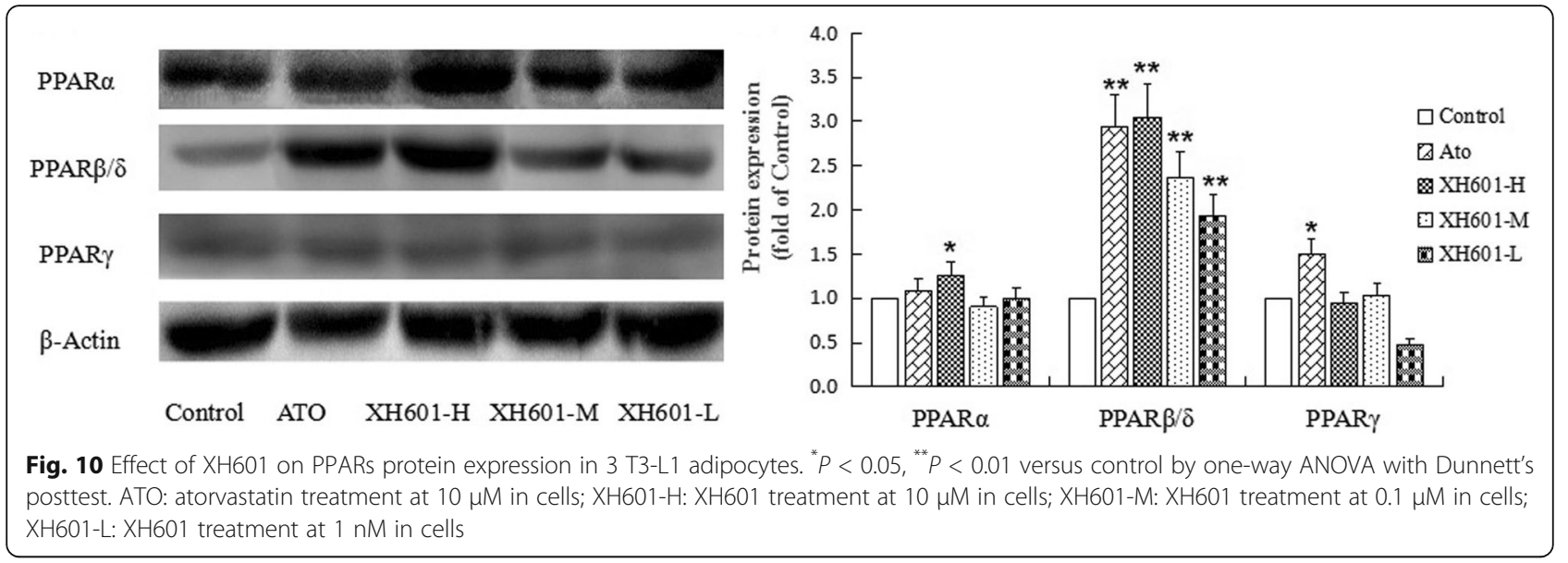

serum LDL-C, TG and hepatic TG levels more potently than atorvastatin. Therefore, XH601 showed stronger hypolipidemic effect when comparing with atorvastatin.

Moreover, there have been several tantalizing clues that $\operatorname{PPAR} \beta / \delta$ may also modulate aspects of lipid homeostasis [39] and it can be proposed as a possible pharmacological target for the treatment of obesity, IR, and dyslipidemia in order to contribute the potent vascular antiatherogenic effects [40]. PPAR $\beta / \delta$ activation inhibits human macrophage foam cell formation and inflammation induced by VLDL and it also exerts favorable effects on HDL-C level [41]. Moreover, relevant study showed that PPAR $\beta / \delta$ agonist treatment decreased plasma TG, Apo-B, total and LDL-cholesterol [42]. Our data suggested that XH601 up-regulated PPAR $\beta / \delta$ almost at the same level as atorvastatin. And XH601 treatment significantly decreased serum TC, Apo-B and increased HDL-C level in hamsters. So the elevated expression of PPAR $\beta / \delta$ had a favorable role in XH601 treatment to combat hyperlipidemia.

The molecular mechanism of the role of PPAR $\gamma$ in adipocytes has been explored as well. PPARY can inhibit the lipolysis by stabilizing the deposited fat with perilipin as well as enhancing the insulin sensitivity
[43] by transcriptionally activating genes involved in insulin signaling, glucose uptake and fatty acid uptake and storage [44]. Nevertheless, heart failure, massive weight gain, and appearance of oedema are relevant to PPAR $\gamma$ [45-47]. It has been shown that moderate reduction of PPAR $\gamma$ activity in mice prevented the insulin resistance and obesity induced by a high-fat diet [48]. In the present experiment, swollen spleen was observed after histological dissection. In addition, kidney percentage with ATO was marked higher than HFD, indicating the kidney-related damage of atorvastatin. By contrast, the hyperlipidemic-associated damages were significantly and dose-dependently optimized in the XH601 groups. The results indicated that appropriate functional antagonism of PPAR $\gamma$ may be a logical approach for protection against obesity and obesity-associated diseases. However, further research should be carried out to testify the favorable effects of XH601 on dyslipidemia.

\section{Conclusions}

The obtained data of the in vivo and in vitro studies suggest that XH601 exhibited strong ability to improve the

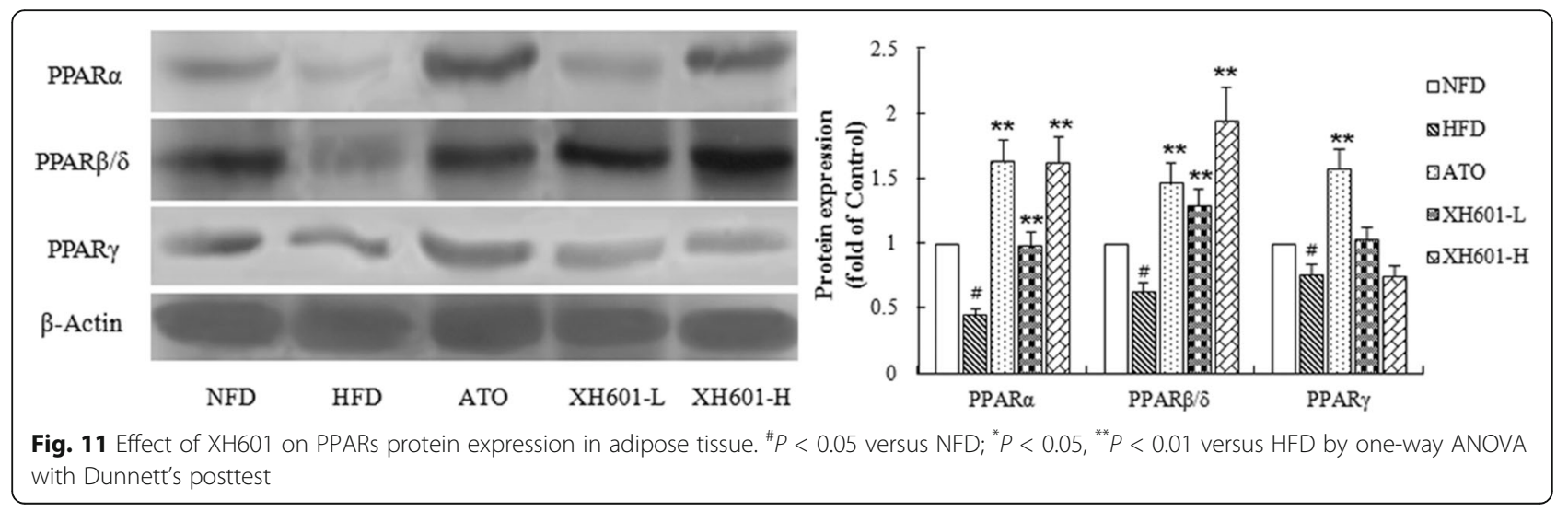


dyslipidemia in hamsters fed with high-fat diet and the hypolipidemic mechanisms of XH601 were associated with the up-regulation of PPAR $\alpha$ and PPAR $\beta / \delta$ expression. The findings in our study supports our hypothesis that XH601 could be developed into a pharmacologic agent for hyperlipidemia with potential hepaticprotective effects on liver damages.

\section{Additional file}

Additional file 1: Figure S12. Effect of $\mathrm{XH} 601$ on phosphorylated PPARa and PPARy protein expression in 3 T3-L1 adipocytes. ${ }^{*} P<0.05$, ${ }^{* *} P<0.01$ versus control by one-way ANOVA with Dunnett's posttest. ATO: atorvastatin treatment at $10 \mu \mathrm{M}$ in cells; XH601-H: XH601 treatment at $10 \mu \mathrm{M}$ in cells: XH601-M: XH601 treatment at $0.1 \mu \mathrm{M}$ in cells; XH601-L: XH601 treatment at $1 \mathrm{nM}$ in cells. Figure S11. Effect of XH601 on phosphorylated PPARa and PPARy protein expression in adipose tissue. ${ }^{\#} P<0.05$ versus NFD; ${ }^{*} P<0.05,{ }^{* *} P<0.01$ versus HFD by one-way ANOVA with Dunnett's posttest. (DOC $217 \mathrm{~kb}$ )

\section{Abbreviations}

ALT: Alanine aminotransferase; Apo-B: Apolipoprotein B; ApoE: Apolipoprotein E; AST: Aspartate aminotransferase; ATO: Atorvastatin; CETP: Cholesteryl ester transfer protein; DMEM: Dulbecco's modified Eagle's medium; EFP: Epididymal fat pad; HDL-C: High-density lipoprotein cholesterol; HED: Human equivalent dose; HFD: High-fat diet; LDL-C: Low-density lipoprotein cholesterol; NFD: Normal-fat diet; PBS: Phosphate-buffered saline; qRT-PCR: Quantitative real time polymerase chain reaction; TC: Total cholesterol; TG: Triglyceride; XH601: 3-(4-methoxyphenyl)7-(undec-10-en-1-yloxy)-4H-benzopyran-4-one

\section{Acknowledgments}

The authors acknowledge Chinese Ministry of Science and Technology supporting this program.

\section{Funding}

This research has been funded by Twelfth 5-Year original new drug research program (2012ZX09103-101-041).

\section{Availability of data and materials}

All data generated or analyzed during this study are included within the article.

\section{Authors' contributions}

$\mathrm{XH}$ conceived and designed the experiments. ZMJ, WSS, JY, WY and SH carried out the laboratory experiments. ZMJ analyzed the data, prepared figures and wrote the manuscript. XH and XP contributed reagents. All authors read and approved the final manuscript.

\section{Competing interests}

The authors declare that they have no competing interests.

\section{Consent for publication}

Not applicable.

\section{Ethics approval and consent to participate}

All animal experiments were approved by the Committee on the Medical Ethics of Nanjing Brain Hospital, affiliated to Nanjing Medical University (Permit Number: 2011KY45), and followed National Research Council Guidelines.

\section{Author details}

${ }^{1}$ Nanjing Medical University, Affiliated Nanjing Brain Hospital, No. 264 Guangzhou Road, Nanjing, Jiangsu 210029, People's Republic of China. ${ }^{2}$ Department of Pharmaceutical Chemistry, China Pharmaceutical University, No. 24 Tong Jia Xiang, Nanjing, China.
Received: 12 January 2017 Accepted: 21 April 2017

Published online: 02 May 2017

\section{References}

1. Beaglehole R, Yach D. Globalisation and the prevention and control of noncommunicable disease: the neglected chronic diseases of adults. Lancet. 2003;362:903-8

2. Dhingra D, Lamba D. Antihyperlipidemic activity of Aloe succotrina in rats: possibly mediated by inhibition of HMG-CoA Reductase. ISRN Pharmacol. 2014; doi:10.1155/2014/243575.

3. Kolovou GD, Anaqnostopoulou KK. Pathophysiology of dyslipidaemia in the metabolic syndrome. Postgrad Med J. 2005;81:358-66.

4. Izadpanah $\mathrm{R}$, Schächtele DJ. The impact of statins on biological characteristics of stem cells provides a novel explanation for their pleiotropic beneficial and adverse clinical effects. Am J Physiol Cell Physiol. 2015:309:C522-31.

5. Bays H. Statin safety: an overview and assessment of the data-2005. Am J Cardiol. 2006:97:6C-26C.

6. Hamann PD, Cooper RG. Statin-induced necrotizing myositis - a discrete autoimmune entity within the "statin-induced myopathy spectrum". Autoimmun Rev. 2013;12:1177-81.

7. Murinson BB, Hauqhey NJ. Selected statins produce rapid spinal motor neuron loss in vitro. BMC Musculoskelet Disord. 2012;13:100.

8. Sattar N, Preiss D. Statins and risk of incident diabetes: a collaborative metaanalysis of randomised statin trials. Lancet. 2010;375:735-42.

9. Tierney EF, Thurman DJ. Association of statin use with peripheral neuropathy in the U.S. population 40 years of age or older. J Diabetes. 2013:5:207-15.

10. Yin J, Zhang $\mathrm{H}$. Traditional Chinese medicine in treatment of metabolic syndrome. Endocr Metab Immune Disord Drug Targets. 2008:8:99-111.

11. Usui T. Pharmaceutical prospects of phytoestrogens. Endocr J. 2006;53:7-20.

12. Patel RP, Barnes S. Isoflavones and PPAR signaling: a critical target in cardiovascular, metastatic, and metabolic disease. PPAR Res. 2010; doi:10.1155/2010/153252

13. Yalniz $M$, Bahcecioglu $H$. Preventive role of genistein in an experimental nonalcoholic steatohepatitis model. J Gastroenterol Hepatol. 2007;22:2009-14.

14. Pakalapati G, Li L. Influence of red clover (Trifolium pratense) isoflavones on gene and protein expression profiles in liver of ovariectomized rats. Phytomedicine. 2009;16:845-55

15. Evans RM. The nuclear receptor superfamily: a rosetta stone for physiology. Mol Endocrinol. 2005:19:1429-38.

16. Shen P, Liu MH. Differential effects of isoflavones, from Astragalus Membranaceus and Pueraria Thomsonii, on the activation of PPARalpha, PPARgamma, and adipocyte differentiation in vitro. J Nutr. 2006;136:899-905.

17. Kan CF, Singh AB. PPAR $\delta$ activation induces hepatic long-chain acyl-CoA synthetase 4 expression in vivo and in vitro. Biochim Biophys Acta. 1851;2015:577-87.

18. Moghadasian MH. Experimental atherosclerosis: a historical overview. Life Sci. 2002;70:855-65.

19. Bishop RW. Structure of the hamster low density lipoprotein receptor gene. J Lipid Res. 1992;33:549-57.

20. Chen W, Fan S. Novel PPAR pan agonist, ZBH ameliorates hyperlipidemia and insulin resistance in high fat diet induced hyperlipidemic hamster. PLoS One. 2014;9:e96056.

21. Sullivan MP, Cerda JJ. The gerbil, hamster, and guinea pig as rodent models for hyperlipidemia. Lab Anim Sci. 1993:43:575-8.

22. Sima A, Stancu C. The hyperlipemic hamster - a model for testing the antiatherogenic effect of amlodipine. J Cell Mol Med. 2001;5:153-62.

23. Lim MK, Ku SK. Effect of polycan, a $\beta$-glucan originating from Aureobasidium, on a high-fat diet-induced hyperlipemic hamster model. Exp Ther Med. 2015;9:1369-78.

24. Huang WC, Chen YM. Hypolipidemic effects and safety of lactobacillus reuteri 263 in a hamster model of hyperlipidemia. Nutrients. 2015;7:3767-82.

25. Chen WC, Huang WC. Whey protein improves exercise performance and biochemical profiles in trained mice. Med Sci Sports Exerc. 2014:46:1517-24.

26. Fishbein MC. The vulnerable and unstable atherosclerotic plaque. Cardiovasc Pathol. 2010;19:6-11.

27. Tenenbaum A, Fisman EZ. Fibrates are an essential part of modern antidyslipidemic arsenal: spotlight on atherogenic dyslipidemia and residual risk reduction. Cardiovasc Diabetol. 2012;11:125. 
28. Corti R, Fuster V. Lipid lowering by simvastatin induces regression of human atherosclerotic lesions: two years' follow-up by high-resolution noninvasive magnetic resonance imaging. Circulation. 2002;106:2884-7.

29. Pucci A, Sheiban I. In vivo coronary plaque histology in patients with stable and acute coronary syndromes: relationships with hyperlipidemic status and statin treatment. Atherosclerosis. 2007;194:189-95.

30. Crisby M, Nordin-Fredriksson G. Pravastatin treatment increases collagen content and decreases lipid content, infammation, metalloproteinases, and cell death in human carotid plaques: implications for plaque stabilization. Circulation. 2001;103:926-33.

31. Wang P, Xu S. Salvianolic acid B inhibited PPARy expression and attenuated weight gain in mice with high-fat diet-induced obesity. Cell Physiol Biochem. 2014;34:288-98.

32. Zou C, Shao J. Role of adipocytokines in obesity-associated insulin resistance. J Nutr Biochem. 2008:19:277-86.

33. Mezei O, Banz WJ. Soy Isoflavones exert Antidiabetic and Hypolipidemic effects through the PPAR pathways in obese Zucker rats and Murine RAW 264.7 cells. J Nutr. 2003;133:1238-43.

34. Michalik L, Auwerx J. International Union of Pharmacology. LXI. Peroxisome Proliferator-activated receptors. Pharmacol Rev. 2006;58:726-41.

35. Han L, Li M. Atorvastatin may delay cardiac aging by upregulating peroxisome proliferator-activated receptors in rats. Pharmacology. 2012;89:74-82.

36. Sheng L, Yang X. Effect of Atorvastatin on expression of Peroxisome Proliferator-activated receptor Beta/delta in Angiotensin II-induced hypertrophic myocardial cells in vitro. Chin Med Sci J. 2015;30:245-51.

37. Zhao J, Cheng Q. Atorvastatin improves pathological changes in the aged kidney by upregulating peroxisome proliferator-activated receptor expression and reducing matrix metalloproteinase-9 and transforming growth factor- $\beta 1$ levels. Exp Gerontol. 2016;74:37-42.

38. Contreras AV, Torres N. PPAR-a as a key nutritional and environmental sensor for metabolic adaption. Adv Nutr. 2013;4:439-52.

39. Oliver WR, Shenk JL. A selective peroxisome proliferator-activated receptor delta agonist promotes reverse cholesterol transport. Proc Natl Acad Sci U S A. 2001:98:5306-11.

40. Ajith TA, Jayakumar TG. Peroxisome proliferator-activated receptors in cardiac energy metabolism and cardiovascular disease. Clin Exp Pharmacol Physiol. 2016;43:649-58.

41. Vázquez-Carrera M. Unraveling the effects of PPAR $\beta / \delta$ on insulin resistance and cardiovascular disease. Trends Endocrinol Metab. 2016;27:319-34.

42. Risérus U, Sprecher D. Activation of peroxisome proliferator-activated receptor (PPAR) delta promotes reversal of multiple metabolic abnormalities, reduces oxidative stress, and increases fatty acid oxidation in moderately obese men. Diabetes. 2008;57:332-9.

43. Khan SA, Sathyanarayan A. ATGL-catalyzed lipolysis regulates SIRT1 to control PGC-1a/PPAR-a signaling. Diabetes. 2015;64:418-26.

44. Brown JM, McIntosh MK. Conjugated Linoleic acid in humans: regulation of adiposity and insulin sensitivity. J Nutr. 2003:133:3041-6.

45. Tang WH, Maroo A. PPARgamma agonists: safety issues in heart failure. Diabetes Obes Metab. 2007:9:447-54.

46. Wright MB, Bortolini M. Minireview: challenges and opportunities in development of PPAR agonists. Mol Endocrinol. 2014;28:1756-68.

47. Jia Z, Sun Y. New insights into the PPARY agonists for the treatment of diabetic nephropathy. PPAR Res. 2014; doi:10.1155/2014/818530.

48. Yamauchi T, Waki H. Inhibition of RXR and PPARgamma ameliorates dietinduced obesity and type 2 diabetes. J Clin Invest. 2001;108:1001-13.

\section{Submit your next manuscript to BioMed Central and we will help you at every step:}

- We accept pre-submission inquiries

- Our selector tool helps you to find the most relevant journal

- We provide round the clock customer support

- Convenient online submission

- Thorough peer review

- Inclusion in PubMed and all major indexing services

- Maximum visibility for your research

Submit your manuscript at www.biomedcentral.com/submit
Biomed Central 